\title{
Special Session on Certification of Dynamic and Adaptive Systems
}

\author{
Paul R. Work ${ }^{1}$ \\ ${ }^{1}$ Raytheon Company \\ Portsmouth, RI 02871 USA \\ paul_r_work@raytheon.com
}

\begin{abstract}
As part of the $15^{\text {th }}$ International Workshop on Parallel and Distributed Real-Time Systems, 26-27 March 2007, Long Beach, CA, to be held in conjunction with IPDPS 2007, there will be a Special Session focused on the topic of Certification of Dynamic and Adaptive Systems.

The verification, validation, and eventual certification of dynamic and adaptive systems is a challenging set of activities both intellectually and, at this time, physically, due to the limits of the state of research and technology in this area. Many more systems are being built using today's dynamic technologies to achieve significant operational capabilities in a timely manner and yet some will need to operate safely and all will need to perform reliably. This is complicated further if these solutions need to do so in a low latency environment with little to no failure cases. The purpose of this session is to bring to light research and development being performed (or even, just being dreamed of) to look at the scalability problems with certifying dynamic and adaptive solutions.
\end{abstract}

\section{Background}

Over the past several years the computing environments in a wide variety of applications, varying from healthcare, to financial, to military, and onto automotive, are making more and more use of distributing computing platforms. So whether this is a medical testing system or an automobile or a military weapon system, these sorts of systems are becoming a part of everyday life. As such, they affect the well being of everyone, therefore correct and safe operation of these systems is necessary. To assure this is happening requires techniques and methods to verify and validate these systems are designed, implemented, and operate as intended. Past IPDPS conferences have covered the topic of certification of distributed real-time systems [1,2]. This Special Session will continue this work in order to keep attention on this vital and important area of computer science.

\section{Overview of the Special Session}

The material to be covered varies from static a priori analysis through to collection and analysis performed while a system (or system of systems) is operating. The topics to be discussed will cover: intelligent instrumentation, statistical analysis, definition, testing, and analysis of operating boundaries and boundedness of solutions.

Current invited presenters come from both academia and industry to bring additional approaches and solutions to this challenge.

The format of the session is intended to incorporate a good deal of interaction among the speakers and participants to foster idea exchange and to make more visible both aspects the problem space and potential new approaches.

\section{References}

[1] Suzhen Lin, A. Sai Sudhir, G. Manimaran, "ConFiRMDRTS: A Certification Framework for Dynamic Resource Management in Distributed Real-Time Systems," IPDPS, p. 110a, International Parallel and Distributed Processing Symposium (IPDPS'03), 2003.

[2] Lonnie R. Welch, "An Approach for On-line Performance Certification," IPDPS, p. 110b, International Parallel and Distributed Processing Symposium (IPDPS'03), 2003. 